\title{
Multi-Basis-Set (TD-)DFT Methods for Predicting Electron Attachment Energies Supporting Information
}

\author{
Guillaume Thiam* and Franck Rabilloud* \\ Université de Lyon, Université Claude Bernard Lyon 1, CNRS, Institut Lumière Matière, \\ UMR5306, F-69622 Villeurbanne, France \\ E-mail: guillaume.thiam@univ-lyon1.fr; franck.rabilloud@univ-lyon1.fr
}

Supporting information:

Table SI1: Excitation energies of the anion calculated at TDDFT/ $\omega$ B97x level using the basis sets cc-pvtz, aug-cc-pvtz, and the stabilization graph method.

Table SI2: Comparison of attachment energies calculated at TDDFT/B3LYP and EOMCCSD levels with experimental results.

Figure SI1: Plot of the energies of virtual orbitals of $\mathrm{CCl}_{2} \mathrm{~F}_{2}$ relative to the scaling factor $\alpha$. Figure SI2: Molecules considered in the present investigation.

Figure SI3: Comparison of the experimental data and the calculated $\omega$ B97x/cc-pvtz values.

Figure SI4: Comparison of the experimental data and the calculated B3LYP/MBS values. Figure SI5: Comparison of the experimental data and the calculated B3LYP/cc-pvtz values.

Figure SI6: Comparison of the experimental data and the calculated values obtained with $\omega \mathrm{B} 97 \mathrm{x} / \mathrm{MBS}$ and EOM-CCSD/MBS.

Coordinates of optimized structures of molecules. 
Tables 
Table SI1: Excitation energies of the anion calculated at TDDFT/ $\omega$ B97x level. ${ }^{a}$ When two values are given for $\omega \mathrm{B} 97 \mathrm{x} /$ aug-cc-pvtz, they correspond to the energies of a valence state and a continuum state which are involved in an avoided crossing visible in the stabilization graph. ${ }^{b}$ The stabilization graph method is performed with the basis set aug-cc-pvtz where the most diffuse exponents are scaled by a factor $\alpha=0.5-3$.

\begin{tabular}{|c|c|c|c|}
\hline molecule & wB97x/cc-pvtz & $\omega$ B97x/aug-cc-pvtz ${ }^{a}$ & Stabilization method $^{b}$ \\
\hline Pyridine & $\begin{array}{l}0.44 \\
3.98\end{array}$ & $\begin{array}{l}0.46 \\
4.05\end{array}$ & \\
\hline Dichlorodifluoromethane & $\begin{array}{l}1.49 \\
3.31\end{array}$ & $\begin{array}{l}0.46 ; 1.05 \\
2.11 ; 3.75\end{array}$ & $\begin{array}{l}1.25 \\
3.35\end{array}$ \\
\hline Chlorobenzene & $\begin{array}{l}0.13 \\
1.60 \\
3.89 \\
4.25\end{array}$ & $\begin{array}{c}0.10 \\
1.41 ; 2.10 \\
3.52 ; 3.79 \\
4.05\end{array}$ & $\begin{array}{l}0.12 \\
2.19 \\
3.79 \\
4.23\end{array}$ \\
\hline Buta-1,3-diene & 2.65 & $1.42 ; 2.21$ & 2.16 \\
\hline Benzonitrile & $\begin{array}{l}0.76 \\
3.04 \\
3.66 \\
4.63\end{array}$ & $\begin{array}{l}0.72 \\
2.75 \\
3.24 \\
4.25\end{array}$ & $\begin{array}{l}0.73 \\
3.01 \\
3.50 \\
4.28\end{array}$ \\
\hline p-benzoquinone & $\begin{array}{l}2.73 \\
3.53 \\
6.18\end{array}$ & $\begin{array}{l}2.67 \\
3.36 \\
6.27\end{array}$ & \\
\hline Benzene & 3.91 & 4.15 & \\
\hline Pyrazine & $\begin{array}{l}0.84 \\
4.05\end{array}$ & $\begin{array}{l}0.90 \\
4.08\end{array}$ & \\
\hline Nitrobenzene & $\begin{array}{l}1.56 \\
2.42 \\
5.12\end{array}$ & $\begin{array}{l}1.65 \\
2.34 \\
5.10\end{array}$ & \\
\hline Phenylacetylene & $\begin{array}{l}0.73 \\
2.66 \\
3.35 \\
4.18\end{array}$ & $\begin{array}{l}0.70 \\
2.65 \\
3.07 \\
4.15\end{array}$ & $\begin{array}{l}0.70 \\
2.46 \\
3.14 \\
4.13\end{array}$ \\
\hline Furan & 1.62 & 1.43 & \\
\hline Nitromethane & 3.97 & 2.77 & 3.33 \\
\hline Water & $\begin{array}{c}6.68 \\
8.75 \\
11.24\end{array}$ & $\begin{array}{l}7.60 \\
9.32 \\
9.46\end{array}$ & $\begin{array}{c}7.07 \\
9.23 \\
11.81\end{array}$ \\
\hline Benzaldehyde & $\begin{array}{l}1.36 \\
2.86 \\
4.21\end{array}$ & $\begin{array}{l}1.31 \\
2.80 \\
4.19\end{array}$ & \\
\hline Phenyl-isothiocyanate & $\begin{array}{l}0.84 \\
2.62 \\
4.15\end{array}$ & $\begin{array}{l}0.79 \\
2.38 \\
4.07\end{array}$ & \\
\hline Butadiyne & 4.45 & 4.64 & 4.38 \\
\hline
\end{tabular}


Table SI1 continued.

\begin{tabular}{|l|c|c|c|}
\hline \hline molecule & $\omega$ B97x/cc-pvtz & $\omega$ B9/aug-cc-pvtz & \\
\hline Cyanogene & 5.30 & 5.19 & 5.24 \\
\hline GeCl $_{4}$ & 2.59 & 2.53 & \\
& 6.87 & 6.67 & \\
\hline \hline
\end{tabular}

Table SI2: Comparison of attachment energies calculated at TDDFT/B3LYP and EOM-CCSD levels with experimental results. EOM-CCSD/MBS means vertical electron affinity (VEA) calculated at $\operatorname{CCSD}(\mathrm{T}) /$ aug-cc-pvtz level, and anionic excitation at EOM-CCSD/cc-pvtz level. EOM-CCSD/cc-pvtz means vertical electron affinity (VEA) calculated at CCSD $(\mathrm{T}) /$ cc-pvtz level, and anionic excitations at EOM-CCSD/cc-pvtz level.

B3LYP/MBS stands for B3LYP/Multi-Basis-Set (TD(cc-pvtz) + VEA (aug-cc-pvtz)).

\begin{tabular}{|l|c|c|c|c|c|}
\hline \hline molecule & $\begin{array}{c}\text { B3LYP } \\
\text { cc-pvtz }\end{array}$ & $\begin{array}{c}\text { B3LYP } \\
\text { MBS }\end{array}$ & $\begin{array}{c}\text { EOM-CCSD } \\
\text { cc-pvtz }\end{array}$ & $\begin{array}{c}\text { EOM-CCSD } \\
\text { MBS }\end{array}$ & Expt \\
\hline pyridine & 1.186 & 0.479 & 1.244 & 1.000 & $0.59^{f}$ \\
$\mathrm{C}_{5} \mathrm{H}_{5} \mathrm{~N}$ & 1.582 & 0.875 & 1.755 & 1.512 & $1.16^{f}$ \\
& 5.149 & 4.442 & 5.474 & 5.230 & $4.48^{f}$ \\
\hline dichlorodifluoromethane & 1.241 & 0.492 & 2.004 & 0.992 & $0.97^{g}$ \\
$\mathrm{CCl}_{2} \mathrm{~F}_{2}$ & 3.009 & 2.261 & 3.584 & 2.572 & $2.36^{g}$ \\
& 4.692 & 3.944 & 5.447 & 4.433 & $3.86^{g}$ \\
\hline chlorobenzene & 1.280 & & 1.555 & 0.703 & $0.75^{h}$ \\
$\mathrm{C}_{6} \mathrm{H}_{5} \mathrm{Cl}$ & 1.395 & & 1.756 & 0.925 & $0.75^{h}$ \\
& 2.847 & & 2.796 & 1.945 & $2.42^{h}$ \\
& 5.069 & & 5.583 & 4.732 & $4.39^{h}$ \\
\hline buta-1,3-diene & 1.155 & 0.748 & 1.298 & 0.786 & $0.62^{e}$ \\
$\mathrm{C}_{4} \mathrm{H}_{6}$ & 3.840 & 3.433 & 3.946 & 3.352 & $2.8^{e}$ \\
\hline benzonitrile & 0.231 & 0.040 & 0.623 & 0.323 & $0.57^{d}$ \\
$\mathrm{C}_{7} \mathrm{H}_{5} \mathrm{~N}$ & 3.723 & 3.539 & 3.738 & 3.437 & $2.57^{d}$ \\
& 4.018 & 3.827 & 4.343 & 4.133 & $3.19^{d}$ \\
& 4.461 & 4.278 & 5.238 & 4.937 & $4.62^{d}$ \\
\hline
\end{tabular}


Table SI2 continued.

\begin{tabular}{|c|c|c|c|c|c|}
\hline molecule & $\begin{array}{l}\text { B3LYP } \\
\text { cc-pvtz }\end{array}$ & $\begin{array}{c}\text { B3LYP } \\
\text { MBS }\end{array}$ & $\begin{array}{c}\text { EOM-CCSD } \\
\text { cc-pvtz }\end{array}$ & $\begin{array}{c}\text { EOM-CCSD } \\
\text { MBS }\end{array}$ & Expt \\
\hline \multirow{3}{*}{$\begin{array}{l}p \text {-benzoquinone } \\
\mathrm{C}_{6} \mathrm{H}_{4} \mathrm{O}_{2}\end{array}$} & 0.863 & 0.692 & & & $0.69^{b}$ \\
\hline & 1.695 & 1.524 & & & $1.41^{b}$ \\
\hline & 4.615 & 4.444 & & & $4.37^{b}$ \\
\hline \multirow{2}{*}{$\begin{array}{l}\text { benzene } \\
\mathrm{C}_{6} \mathrm{H}_{6}\end{array}$} & 1.750 & 0.445 & & & $1.13^{c}$ \\
\hline & 5.568 & 4.263 & & & $4.84^{c}$ \\
\hline \multirow{3}{*}{$\begin{array}{l}\text { pyrazine } \\
\mathrm{C}_{4} \mathrm{H}_{4} \mathrm{~N}_{2}\end{array}$} & 0.543 & 0.214 & & & $0.07^{a}$ \\
\hline & 1.305 & 0.975 & & & $0.87^{a}$ \\
\hline & 4.611 & 3.444 & & & $4.1^{a}$ \\
\hline \multirow{3}{*}{$\begin{array}{l}\text { nitrobenzene } \\
\mathrm{C}_{6} \mathrm{H}_{5} \mathrm{NO}_{2}\end{array}$} & 0.624 & 0.362 & & & $0.55^{j}$ \\
\hline & 1.945 & 1.683 & & & $1.36^{j}$ \\
\hline & 4.415 & 4.153 & & & $4.69^{j}$ \\
\hline \multirow{5}{*}{$\begin{array}{l}\text { phenylacetylene } \\
\mathrm{C}_{8} \mathrm{H}_{6}\end{array}$} & 0.711 & 0.455 & & & $0.35^{i}$ \\
\hline & 1.373 & 1.117 & & & $1.03^{i}$ \\
\hline & 2.739 & 2.482 & & & $2.41^{i}$ \\
\hline & 3.901 & 3.644 & & & $3.28^{i}$ \\
\hline & 4.881 & 4.625 & & & $4.83^{i}$ \\
\hline \multirow{2}{*}{$\begin{array}{l}\text { Furan } \\
\mathrm{C}_{4} \mathrm{H}_{4} \mathrm{O}\end{array}$} & 2.377 & 0.559 & & & $1.73^{l}$ \\
\hline & 3.988 & 2.170 & & & $3.15^{l}$ \\
\hline \multirow{2}{*}{$\begin{array}{l}\text { nitromethane } \\
\mathrm{CH}_{3} \mathrm{NO}_{2}\end{array}$} & 0.719 & 0.017 & & & $0.45^{j}$ \\
\hline & 4.048 & 3.346 & & & $4.0^{j}$ \\
\hline \multirow{3}{*}{$\begin{array}{l}\text { water } \\
\mathrm{H}_{2} \mathrm{O}\end{array}$} & 9.113 & 6.645 & & & $6.9^{k}$ \\
\hline & 10.759 & 8.291 & & & $8.9^{k}$ \\
\hline & 14.177 & 11.709 & & & $11.8^{k}$ \\
\hline \multirow{3}{*}{$\begin{array}{l}\text { Benzaldehyde } \\
\mathrm{C}_{7} \mathrm{H}_{6} \mathrm{O}\end{array}$} & 1.083 & 0.883 & & & $0.85^{m}$ \\
\hline & 2.839 & 2.640 & & & $2.21^{m}$ \\
\hline & 4.162 & 3.962 & & & $4.34^{m}$ \\
\hline \multirow{4}{*}{$\begin{array}{l}\text { Phenyl isothiocyanate } \\
\mathrm{C}_{7} \mathrm{H}_{5} \mathrm{NS}\end{array}$} & 0.259 & 0.074 & & & $0.2^{n}$ \\
\hline & 0.946 & 0.761 & & & $0.62^{n}$ \\
\hline & 2.909 & 2.724 & & & $2.23^{n}$ \\
\hline & 4.372 & 4.187 & & & $4.2^{n}$ \\
\hline \multirow{2}{*}{$\begin{array}{l}\text { Butadiyne } \\
\mathrm{C}_{4} \mathrm{H}_{2}\end{array}$} & 1.237 & & 1.892 & 0.663 & $1.0^{\circ}$ \\
\hline & 5.603 & & 6.312 & 5.084 & $5.6^{\circ}$ \\
\hline \multirow{2}{*}{$\begin{array}{l}\text { Cyanogen } \\
\mathrm{C}_{2} \mathrm{~N}_{2}\end{array}$} & -0.046 & -0.261 & 0.564 & 0.307 & $0.58^{\circ}$ \\
\hline & 4.974 & 4.759 & 5.883 & 5.625 & $5.37^{\circ}$ \\
\hline \multirow{3}{*}{$\begin{array}{l}\text { germanium tetrachloride } \\
\mathrm{GeCl}_{4}\end{array}$} & -0.854 & -1.09 & & & $0.0^{p}$ \\
\hline & 2.069 & 1.824 & & & $1.4^{p}$ \\
\hline & 5.523 & 5.278 & & & $5.5^{p}$ \\
\hline
\end{tabular}

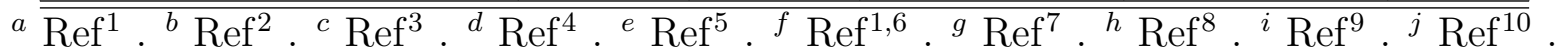
${ }^{k} \operatorname{Ref}^{11} \cdot{ }^{l} \operatorname{Ref}^{12,13} \cdot{ }^{m} \operatorname{Ref}^{14} \cdot{ }^{n} \operatorname{Ref}^{15} \cdot{ }^{o} \operatorname{Ref}^{16} \cdot{ }^{p} \operatorname{Ref}^{17}$. 
Figures

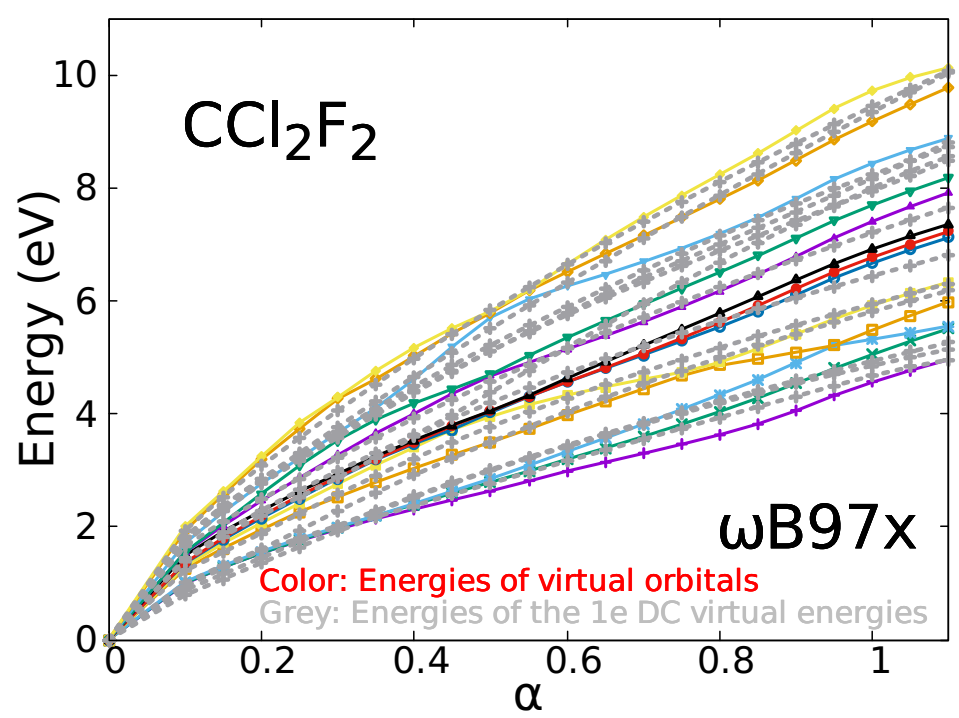

Figure SI1: Plot of the energies of virtual orbitals of $\mathrm{CCl}_{2} \mathrm{~F}_{2}$ relative to the scaling factor $\alpha$. Grey lines are the energies of one free electron in the absence any charges (nuclear and electrons) calculated at the same level of calculation ( $\omega$ B97x) with the same molecular basis set as that used for the molecule. The clear correspondence between energies shows that virtual orbitals of the molecule represent some discretized continuum states (as a "free" electron in a box) when the basis set is enough extended (i.e. small values of $\alpha$ ). 


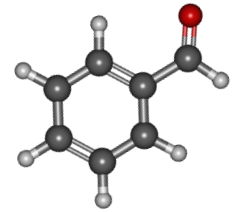

benzaldehyde

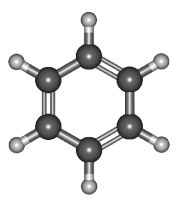

benzene

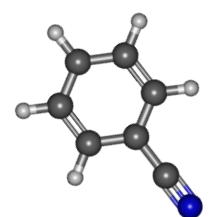

benzonitrile

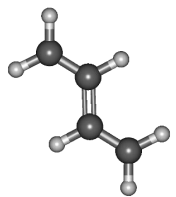

buta-1,3-diene

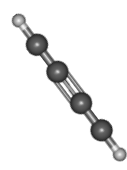

butadiyne

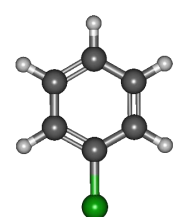

chlorobenzene

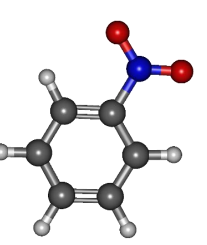

nitrobenzene

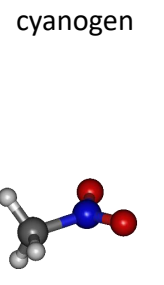

nitromethane

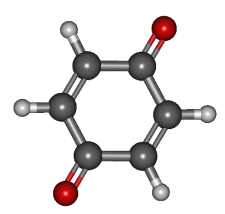

$p$-benzoquinone

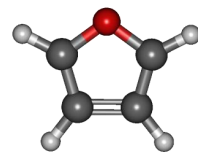

$g_{0}$

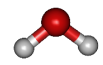

$\mathrm{GeCl}_{4}$

water

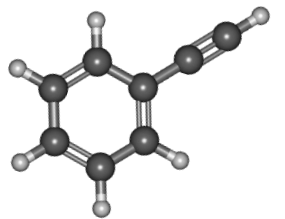

phenylacetylene

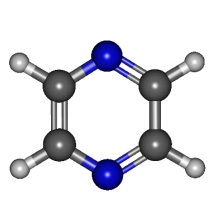

pyrazine

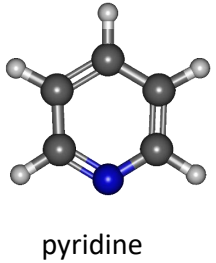

Figure SI2: Molecules considered in the present investigation. 


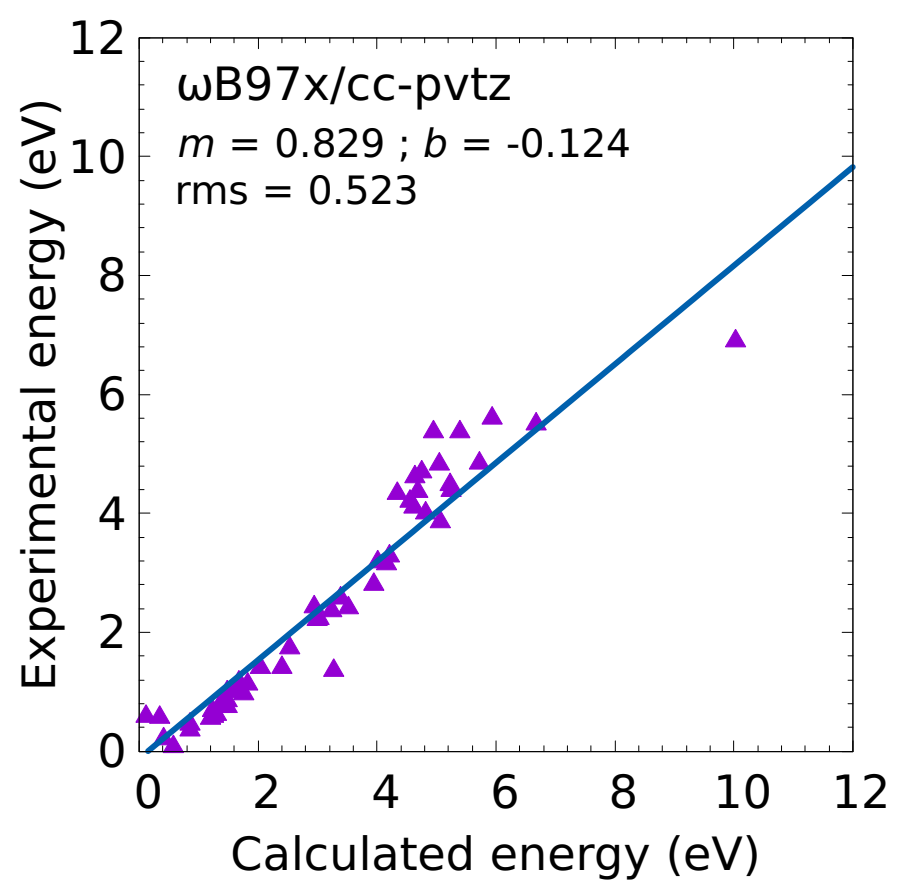

Figure SI3: Comparison of the experimental data and the calculated $\omega \mathrm{B} 97 \mathrm{x} / \mathrm{cc}-\mathrm{pvtz}$ values. 


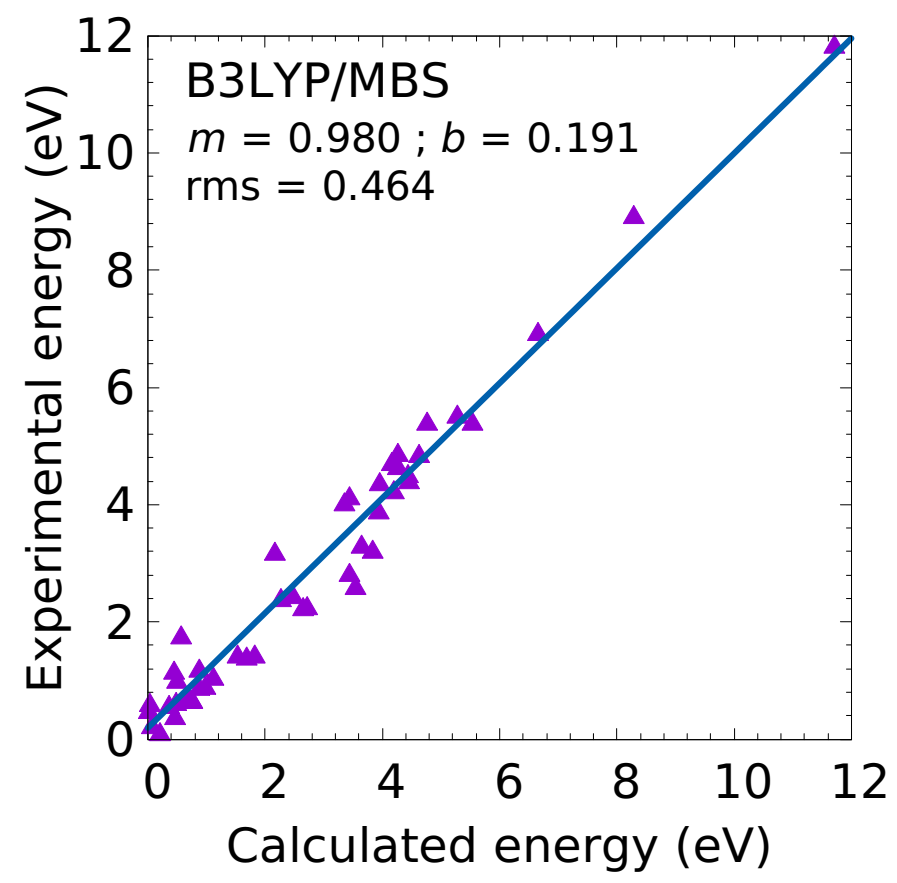

Figure SI4: Comparison of the experimental data and the calculated B3LYP/MBS values.

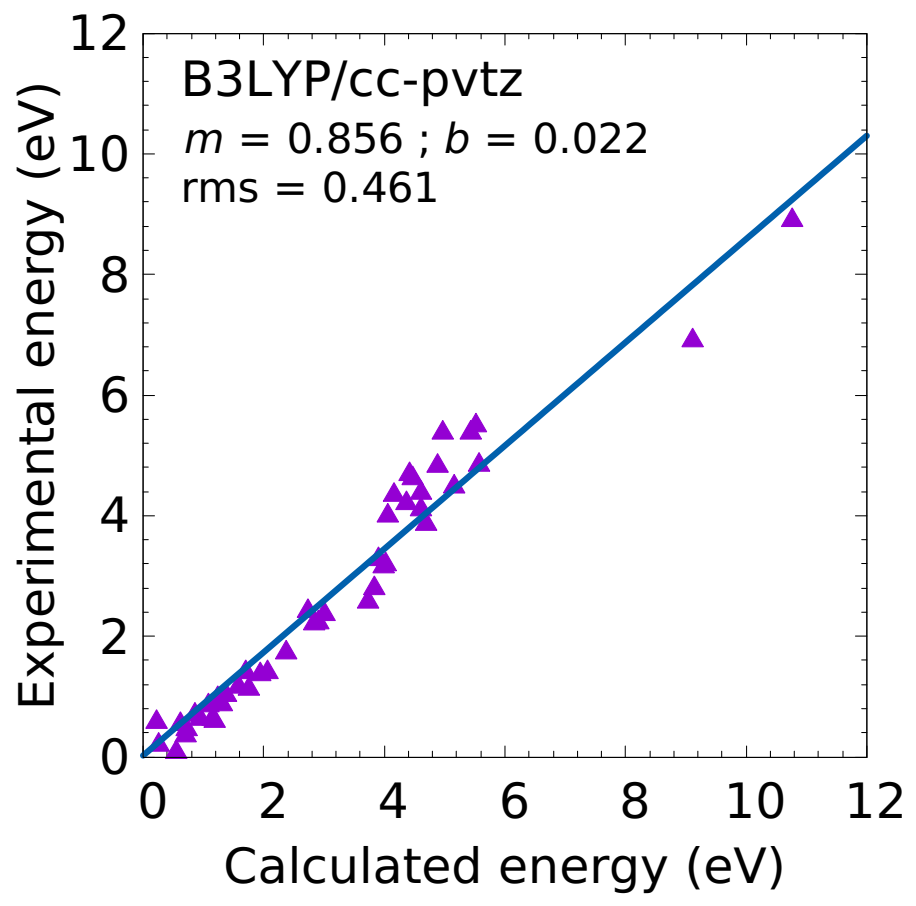

Figure SI5: Comparison of the experimental data and the calculated B3LYP/cc-pvtz values. 


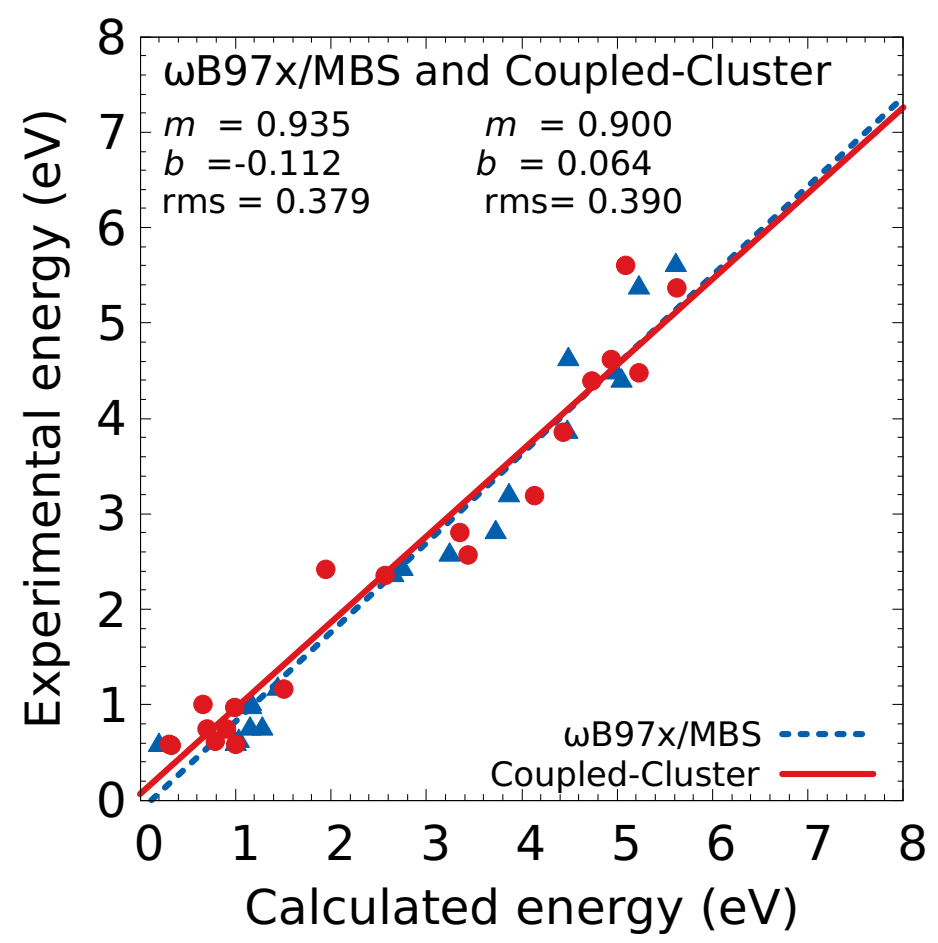

Figure SI6: Comparison of the experimental data and the calculated value obtained with $\omega \mathrm{B} 97 \mathrm{x} / \mathrm{MBS}$ and EOM-CCSD/MBS (electron affinity (VEA) calculated at CCSD(T)/augcc-pvtz level, and anionic excitations at EOM-CCSD/cc-pvtz level). 


\section{References}

(1) Nenner, I.; Schulz, G. J. Temporary negative ions and electron affinities of benzene and N-heterocyclic molecules: pyridine, pyridazine, pyrimidine, pyrazine, and s-triazine. $J$. Chem. Phys. 1975, 62, 1747-1758.

(2) Modelli, A.; Burrow, P. D. Electron transmission study of the negative ion states of p-benzoquinone, benzaldehyde, and related molecules. J. Phys. Chem. 1984, 88, 35503554 .

(3) Burrow, P. D.; Michejda, J. A.; Jordan, K. D. Electron transmission study of the temporary negative ion states of selected benzenoid and conjugated aromatic hydrocarbons. J. Chem. Phys. 1987, 86, 9-24.

(4) Burrow, P. D.; Howard, A. E.; Johnston, A. R.; Jordan, K. D. Temporary anion states of hydrogen cyanide, methyl cyanide, and methylene dicyanide, selected cyanoethylenes, benzonitrile, and tetracyanoquinodimethane. J. Phys. Chem. 1992, 96, 7570-7578.

(5) Burrow, P.; Jordan, K. On the electron affinities of ethylene and 1,3-butadiene. Chemical Physics Letters 1975, 36, 594-598.

(6) Burrow, P. D.; Ashe, A. J.; Bellville, D. J.; Jordan, K. D. Temporary anion states of phosphabenzene, arsabenzene, and stibabenzene. Trends in the $\pi$ and $\pi^{*}$ orbital energies. J. Am. Chem. Soc. 1982, 104, 425-429.

(7) Aflatooni, K.; Burrow, P. Dissociative electron attachment in chlorofluoromethanes and the correlation with vertical attachment energies. Int. J. Mass Spectrom. 2001, 205, $149-161$.

(8) Modelli, A.; Venuti, M. Temporary $\pi^{*}$ and $\sigma^{*}$ Anions and Dissociative Electron Attachment in Chlorobenzene and Related Molecules. J. Phys. Chem. A 2001, 105, 5836-5841. 
(9) Scheer, A. M.; Burrow, P. D. $\pi^{*}$ Orbital System of Alternating Phenyl and Ethynyl Groups: Measurements and Calculations. J. Phys. Chem. B 2006, 110, 17751-17756.

(10) Modelli, A.; Venuti, M. Empty level structure and dissociative electron attachment in gas-phase nitro derivatives. Int. J. Mass Spectrom. 2001, 205, 7-16.

(11) Fedor, J.; Cicman, P.; Coupier, B.; Feil, S.; Winkler, M.; Głuch, K.; Husarik, J.; Jaksch, D.; Farizon, B.; Mason, N. J.; Scheier, P.; Märk, T. D. Fragmentation of transient water anions following low-energy electron capture by H2O/D2O. J. Phys. B: At. Mol. Opt. Phys. 2006, 39, 3935-3944.

(12) Sulzer, P.; Ptasinska, S.; Zappa, F.; Mielewska, B.; Milosavljevic, A. R.; Scheier, P.; Märk, T. D.; Bald, I.; Gohlke, S.; Huels, M. A.; Illenberger, E. Dissociative electron attachment to furan, tetrahydrofuran, and fructose. J. Chem. Phys. 2006, 125.

(13) Modelli, A.; Burrow, P. D. Electron Attachment to the Aza-Derivatives of Furan, Pyrrole, and Thiophene. J. Phys. Chem. A 2004, 108, 5721-5726.

(14) Ameixa, J.; Arthur-Baidoo, E.; da Silva, J. P.; Ryszka, M.; Carmichael, I.; Cornetta, L. M.; do N. Varella, M. T.; da Silva, F. F.; Ptasińska, S.; Denifl, S. Formation of resonances and anionic fragments upon electron attachment to benzaldehyde. Phys. Chem. Chem. Phys. 2020, 22, 8171-8181.

(15) Pshenichnyuk, S. A.; Rakhmeyev, R. G.; Asfandiarov, N. L.; Komolov, A. S.; Modelli, A.; Jones, D. Can the Electron-Accepting Properties of Odorants Be Involved in Their Recognition by the Olfactory System? J. Phys. Chem. Lett. 2018, 9, 2320-2325.

(16) Ng, L.; Balaji, V.; Jordan, K. Measurement of the vertical electron affinities of cyanogen and 2,4-hexadiyne. Chemical Physics Letters 1983, 101, 171-176.

(17) Kumar, T. P. R.; Brynjarsson, B.; Òmarsson, B.; Hoshino, M.; Tanaka, H.; LimãoVieira, P.; Jones, D.; Brunger, M. J.; Ingòlfsson, O. Negative ion formation through 
dissociative electron attachment to the group IV tetrachlorides: Carbon tetrachloride, silicon tetrachloride and germanium tetrachloride. Int. J. Mass Spectrom. 2018, 426, $12-28$. 
xyz coordinates of all molecules.

Benzene

C - 1.2015510 .6934710 .000000

C $0.0000001 .387223-0.000000$

C -1.201456 -0.6936140.000000

C $1.201449-0.693639-0.000000$

C $1.2013590 .693790-0.000000$

C $0.000193-1.3872270 .000000$

H -2.139134 1.2350630 .000000

H $0.000026-2.4699860 .000000$

H $2.1390731 .235146-0.000000$

H -2.138943 -1.2353720.000000

H -0.000234 2.469986-0.000000

H 2.139248 -1.234860 -0.000000

P-Benzoquinone

C -1.2692870.664550 -0.000000

C $-0.0000081 .436831-0.000000$

C -1.269281-0.6644870.000000

C $1.269308-0.6645520 .000000$

C $1.2693080 .664492-0.000000$

C $-0.000008-1.4368340 .000000$

H -2.178028 $1.252902-0.000000$

H $2.1781031 .252781-0.000000$

H -2.178082 -1.252768 0.000000

H 2.178044 -1.252916 0.000000

O $-0.000040-2.6464170 .000000$ 
O $0.0000112 .646417-0.000000$

Benzonitrile

C -1.208460 -0.090670 0.000000

C 0.0000000 .6009700 .000000

C -1.203394 -1.474555 0.000000

C $1.202746-1.4749540 .000000$

C $1.208254-0.0910630 .000000$

C -0.000440 -2.1658290.000000

C 0.0004532 .0384020 .000000

H -2.139583 0.4598560 .000000

H -0.000656 -3.248151 0.000000

H 2.1395350 .4591970 .000000

H -2.140722 -2.014881 0.000000

H $2.139910-2.0155550 .000000$

N 0.0009373 .1865330 .000000

Buta-1,3-diene

C $1.101969-1.4702780 .000000$

C -0.000082 -0.730451 0.000000

C -0.000000 $0.730247-0.000000$

C -1.101933 $1.470445-0.000000$

H 2.084991 -1.013269 0.000000

H $1.060264-2.5510730 .000000$

H -0.973533 -1.212582 0.000000 
H $0.9735881 .212142-0.000000$

H -2.085098 $1.013727-0.000000$

H -1.059940 2.551277-0.000000

Pyridine

C 0.0712131 .3630710 .000000

C 1.2128090 .5764950 .000000

C $0.000000-1.3428080 .000000$

C -1.200656 -0.649305 0.000000

C -1.162910 0.7348170.000000

H 0.1530112 .4416950 .000000

H -2.139629 -1.186384 0.000000

H 2.1953601 .0370650 .000000

H $0.005494-2.4279550 .000000$

H -2.078101 1.3133720 .000000

N $1.191590-0.7530600 .000000$

Nitrobenzene

C $0.000676-2.5008230 .000000$

C -1.203613 -1.8113510.000000

C $1.204800-1.8107550 .000000$

C -0.0000000 .2384890 .000000$

C -1.211826 -0.426380 0.000000

C $1.212263-0.4259590 .000000$

H $0.000963-3.5831810 .000000$ 
H 2.1310110 .1415800 .000000

H -2.131022 0.1403550 .000000

H $2.141630-2.3516170 .000000$

H -2.140302 -2.352459 0.000000

N -0.000512 1.7154470 .000000

O -1.073913 2.275700 0.000000

O 1.0723512 .2765330 .000000

nitromethane

C $0.039005-1.3135410 .000000$

N 0.0000000 .1734180 .000000

O 1.0552790 .7638530 .000000

O - 1.0962110 .6850870 .000000

H -0.490769 -1.647326 0.887216

H $1.074971-1.6295450 .000000$

H -0.490769 -1.647326 -0.887216

Benzaldehyde

C $0.583599-2.1390080 .000000$

C -0.741064 -1.715535-0.000000

C $1.616162-1.2138380 .000000$

C 0.0000000 .5669860 .000000

C -1.033951 -0.364886-0.000000

C 1.3219050 .1407270 .000000

H $0.809829-3.1978690 .000000$ 
H 2.1203430 .8748500 .000000

H -2.055656-0.006759-0.000000

H $2.645517-1.5478230 .000000$

H - $1.541255-2.444318-0.000000$

C -0.299104 2.017299-0.000000

O -1.407149 2.487014-0.000000

H 0.5931252 .6753400 .000000

Butadyine

C 0.0000000 .0000001 .887688

C 0.0000000 .0000000 .690021

C $0.0000000 .000000-0.690021$

C $0.0000000 .000000-1.887688$

H 0.0000000 .0000002 .951266

H $0.0000000 .000000-2.951266$

Cyanogene

C 0.0000000 .0000000 .693935

C $0.0000000 .000000-0.693935$

N 0.0000000 .0000001 .840605

N $0.0000000 .000000-1.840605$

Phenylacetylene (C8H6)

C -0.0000000 .5834840 .000000$

C -1.203973 -0.119978 0.000000 

C 1.204027 -0.119779 0.000000
C $0.000195-2.1989310 .000000$
C -1.200658 -1.504258 0.000000
C 1.200909 -1.504101 0.000000
C -0.000179 2.0189370.000000
C -0.0002913.2164190.000000
H -0.000796 4.2796430.000000
H $2.139741-2.0427480 .000000$
H -2.139387 -2.043083 0.000000

H 2.1368800 .4285380 .000000

H -2.136874 0.428256 0.000000

H $0.000255-3.2813610 .000000$

Pyrazine

C -0.0000001 .3222520 .000000$

C 1.1809530 .5947580 .000000

C $0.000150-1.322257-0.000000$

C -1.181023 -0.594627-0.000000

H 0.0154712 .4063980 .000000

H -2.142222 -1.096366 -0.000000

H 2.1422441 .0962770 .000000

H -0.015553 -2.406418 -0.000000

N -1.189629 $0.732794-0.000000$

N $1.189569-0.7328860 .000000$

Dichlorodifluromethane

C $0.0000030 .337152-0.000012$ 
Cl -1.455130 -0.651288 -0.000035

Cl $1.455134-0.651283-0.000044$

F 0.0000071 .1176961 .075261

F -0.000016 $1.117949-1.075102$

Chlorobenzene

C $0.000865-2.256274-0.000000$

C -1.199166 -1.561087 -0.000000

C $1.200177-1.560497-0.000000$

C -0.0000000 .5031980 .000000$

C -1.207034-0.174561-0.000000

C $1.207176-0.1736390 .000000$

H $0.000989-3.338360-0.000000$

H 2.1359590 .3798090 .000000

H -2.135957 $0.378650-0.000000$

H $2.140180-2.097013-0.000000$

H -2.138621 -2.098563 -0.000000

Cl -0.000862 2.241920 0.000000

Water

O 0.0000000 .0000000 .117043

H $0.0000000 .757944-0.468173$

H $0.000000-0.757944-0.468173$

Furan

C $0.690922-0.9703060 .000000$

C -0.657659-0.9314730.000000 
C 1.1242010 .3943080 .000000

C 0.0000001 .1401500 .000000

O -1.0979020.3484540.000000

H 1.305485 -1.854614 0.000000

H -0.183990 2.200465 0.000000

H -1.419343 -1.691802 0.000000

H 2.1362800 .7622430 .000000

Phenyl-isothiocyanate

C 3.1800750 .2623260 .000132

C $2.689691-1.0350180 .000165$

C 2.2984471 .3339350 .000096

C $0.448917-0.1886460 .000122$

C $1.324800-1.2671630 .000163$

C 0.9317661 .1167150 .000093

H 4.2475250 .4389220 .000135

Н 0.2340151 .9434950 .000066

H $0.926255-2.2724280 .000192$

H 2.6763332 .3480280 .000071

H $3.373295-1.8738750 .000193$

N -0.916289 -0.423953 0.000119

C -2.064314 -0.156006 -0.000138

S -3.618731 $0.124167-0.000331$

\section{GeCl4}

Ge -0.000101 $0.000027-0.000064$

Cl -1.621947 $1.114832-0.767542$ 
Cl -0.711916 -1.824293 0.792143

Cl 0.9549431 .1054651 .525845

Cl $1.379109-0.396054-1.550325$ 